\title{
A CLOSURE PROPERTY OF SETS OF VECTORS
}

\section{BY \\ ROBERT STEINBERG}

1. Introduction and statement of results. If the system $S$ of roots of a simple Lie algebra over the complex field is imbedded in a real Euclidean space in the usual way, the following important property, which we call property $P$, is true:

$$
\text { If } x, y \in S, x \neq-y \text { and }(x, y)<0 \text {, then } x+y \in S ; \quad 0 \notin S .
$$

Our aim here is to study this property. The exclusion of the vector 0 is not essential in what follows. It turns out that many of the other properties of root systems of Lie algebras depend only on property $\mathrm{P}$ and are thus shared by a much wider class of vector systems. In the statements to follow it is assumed that all vectors considered come from a real Euclidean space $V$ of finite dimension $n$.

1.1 Let $S$ be finite and have property $\mathrm{P}$, and let $A$ be a subset with property $\mathrm{P}$. For each $x$ in $S$, assume that at most one (resp. exactly one, at least one) of $x$ and $-x$ is in $A$. Then there is an ordering of the space $V$ such that $A$ is contained in (resp. $A$ is, $A$ contains) the set of positive elements of $S$.

Harish-Chandra [6, Lemma 4] proves the second part of this result for Lie algebra root systems, however, using nontrivial properties of Lie algebras. Borel and Hirzebruch [2, pp. 471-473] give a geometric proof of the second and third parts, but then revert to Lie algebra techniques to prove the first part, all for Lie algebra root systems. All of these authors make a somewhat stronger assumption on $A$ than property $\mathrm{P}$, namely, if $x, y \in A$ and $x+y \in S$, then $x+y \in A$. Our proof of 1.1 depends on a preliminary result which may have some independent interest.

1.2. Let $B$ be finite and have property $\mathrm{P}$, and assume that $x \in B$ implies $-x \notin B$. Then there is an ordering of the space $V$ such that all elements of $B$ are positive.

The real numbers of the form $k-l \sqrt{ } 2$ ( $k, l$ positive integers) show that the assumption of finiteness in 1.1 (or 1.2) can not be dropped. It can, however, be weakened thus.

$1.1^{\prime}$. In 1.1 replace the assumption of finiteness by the assumption that 0 is not a point of accumulation of $S$.

In 1.2 we can go further, in terms of a weakening of property $P$.

$\left(\mathrm{P}_{n}\right) \quad$ If $x, y \in S, x \neq-y$ and $(x, y) \leqq-1 / n|x||y|$, then $x+y \in S ; \quad 0 \notin S$.

Received by the editors September 15, 1961. 
1.2'. If $B$ does not have 0 as a point of accumulation, if $B$ has property $P_{n}$, and if $x \in B$ implies $-x \notin B$, the conclusion of 1.2 holds.

In this statement the number $-1 / n$ which enters via $P_{n}$ can not be replaced by a smaller one, as we see by taking $B$ to be the set of vertices of a regular simplex. We also remark that the change $P$ to $P_{n}$ renders false the third part of $1.1^{\prime}$ as well as all of the results to follow.

We call a set of vectors $S$ symmetric if $x \in S$ implies $-x \in S$, and indecomposable if it is not contained in the union of two lower-dimensional orthogonal subspaces of $V$. Observe that if $S$ is indecomposable then it generates $V$.

1.3. Let $S$ be finite, symmetric and have property $P$, and relative to a fixed ordering of $V$, let $B$ consist of those positive elements of $S$ which can not be written as sums of other positive elements of $S$. Then (a) $B$ is linearly independent; (b) every positive element of $S$ is a sum of elements of $B$. If further $S$ is indecomposable (so that $B$ is a basis of $V$ ), then (c) $B$ cannot be split into subsets $B_{1}, B_{2}$ such that $x_{1} \in B_{1}, x_{2} \in B_{2}$ implies $x_{1}+x_{2} \notin S$; (d) the sum of all elements of $B$ is in $S$; (e) there exists $a$ (dominant) positive element $d$ of $S, d=\sum k(b) b$ $\left(b \in B, k(b)=\right.$ nonnegative integer), such that if $r=\sum l(b) b$ is any element of $S$ then $l(b) \geqq l(b)$ for all $b$.

The proofs of these results can be patterned after those of Cartan [3] and Dynkin [4, p. 106], and are not given here. The existence of the dominant element $d$ is easily seen to be equivalent to the fact that the inequalities $(d, x)<1$ and $(b, x)>0(b \in B)$ define a simplex which is not pierced by any of the hyperplanes $(r, x)=$ integer $(r \in S)$. More generally, we show:

1.4. Let $S$ be finite, symmetric, indecomposable and have property $\mathrm{P}$. Then the regions into which $V$ is partitioned by the hyperplanes $(r, x)=k(r \in S, k=0$, $\pm 1, \pm 2, \ldots)$ are all simplexes.

An example which yields a nice pattern of triangles of various sizes and shapes (in contrast to the Lie algebra case when all regions are congruent under a discrete group generated by reflections) is obtained by letting $S$ consist of the vectors $( \pm 3,0),(0, \pm 3),(i, j)$, with $i, j$ integers, $|i| \leqq 2,|j| \leqq 2,(i, j) \neq(0,0)$ (coordinates relative to an orthonormal basis of $V$ ). Another example, $S=\{ \pm(1,0), \pm(0,1), \pm(1,1), \pm(1 / 2,-1 / 2)\}$, shows that property $\mathrm{P}$ is not necessary for the conclusion of 1.4 and raises the interesting question: what condition is?

One can easily classify the one-dimensional finite symmetric indecomposable sets with property $\mathrm{P}: S=\{ \pm r, \pm 2 r, \ldots, \pm k r ; r \neq 0, k$ an arbitrary positive integer $\}$. In higher dimensions, however, arbitrarily large multiples can not occur if the set is to remain finite.

1.5. Let $S$ be symmetric, indecomposable, and have property $\mathrm{P}$ and dimension $n \geqq 2$. If $r$ and $5 r$ are in $S$, then $S$ is infinite, in fact all nonzero integral multiples 
of $r$ are in $S$. If it is assumed instead that $r$ and $4 r$ are in $S$, then $S$ need not be infinite.

But for infinite sets of the type under consideration there is a rather drastic consequence.

1.6. Let $S$ be infinite, symmetric, indecomposable, and have property $\mathrm{P}$ and dimension $n \geqq 2$. Then $S \cup\{0\}$ is a group (under the addition of $V$ ).

Combining 1.5 and 1.6 , we get:

1.7. If $S$ is symmetric, indecomposable, has property $\mathrm{P}$ and dimension $n \geqq 2$, and if $S$ contains $r$ and $5 r$, then $S \cup\{0\}$ is a group.

A second consequence of 1.6 is a characterization of vector lattices.

1.8. If $0 \notin S$, then $S \cup\{0\}$ is an $n$-dimensional lattice in $V$ if and only if $S$ is infinite, symmetric, indecomposable, has property $\mathrm{P}$, and does not have 0 as a point of accumulation.

A third consequence is that there is no infinite analogue of 1.4. In order to refine 1.7 (see 4.1), we require a final basic result.

1.9. Let $S$ be symmetric, indecomposable and have property $\mathrm{P}$. Let $s$ be in $S$, and let $S_{1}$ be the nonzero part of the projection of $S$ on $s^{0}$, the orthogonal complement of s. Then $S_{1}$ is symmetric, indecomposable and has property $\mathrm{P}$.

2. Proof of 1.1, 1.2, 1.1' and 1.2'. The deductions of 1.1 from 1.2 and of $1.1^{\prime}$ from $1.2^{\prime}$ are identical and as follows. The choice $B=A$ in 1.2 yields the first part of 1.1 and half of the second part. To get the other half, observe that if $x \in S$, and $x \notin A$, then in turn $-x \in A,-x$ is positive, and $x$ is negative. To prove the third part, we choose $B$ as the complement of $A$ in $S$. Then $B$ has property $\mathrm{P}$ : the assumptions $x \in B, y \in B, x \neq-y,(x, y)<0, x+y \notin B$ imply $x+y \in A,-x \in A,-y \in A$, either $(-x, x+y)<0$ or $(-y, x+y)<0$, so that either $(-x)+(x+y)=y$ is in $A$ or $(-y)+(x+y)=x$ is in $A$, a contradiction. Further $x \in B$ implies $-x \notin B$. Thus by 1.2 there is an ordering of $V$ in which all elements of $B$ are positive. If this ordering is reversed, the vectors of $B$ become negative, and $A$ contains all positive elements of $S$ as required.

To establish 1.2 , we first show that there is a nonzero vector $z$ such that $(z, x) \geqq 0$ for all $x$ in $B$. If this is false, then because $B$ has property $P$, there is a sequence $x_{1}, x_{2}, \ldots$ of elements of $B$ such that $x_{1}+x_{2}, x_{1}+x_{2}+x_{3}, \ldots$ are also in $B$. Since $B$ is finite, two of these sums must be equal, so that a nontrivial relation $\sum y_{j}=0\left(y_{j} \in B\right)$ exists. Among all such relations, pick a shortest one. Its length is at least 3. Now $\left(y_{1}, y_{2}+y_{3}+\ldots\right)=\left(y_{1},-y_{1}\right)<0$, so that $\left(y_{1}, y_{j}\right)<0$ for some $j$, and $y_{1}+y_{j} \in B$. But then the relation can be shortened, a contradiction. This proves our assertion, from which we quickly deduce 1.2 by induction on the dimension of $V$. The result being clear if this dimension is 0 , assume it is positive. Then by the inductive assumption there is an ordering of $z^{0}$, the orthogonal complement of $z$, such that all elements of $z^{0} \cap B$ are positive. We extend this ordering (lexicographically) to $V$ by defining $x$ to be positive if in the re- 
presentation $x=k z+u\left(k\right.$ scalar, $\left.u \in z^{0}\right)$ either $k$ is positive or $k$ is 0 and $u$ is positive. Clearly all elements of $B$ are now positive.

Our proof of $1.2^{\prime}$ requires a preliminary result, proved in two steps.

2.1 If 0 is in the convex closure of $a$ set $B$ of unit vectors, then $(x, y) \leqq-1 / n$ for some $x, y$ in $B$.

By $\left[1\right.$, p. 9], there is a relation $\sum_{j=1}^{m} c_{j} x_{j}=0\left(c_{j}>0, x_{j} \in B, m \leqq n+1\right)$. If $c_{1}$ is the largest of the $c_{j}$, and 2.1 is false, we have $0=c_{1}\left(x_{1}, x_{1}\right)+\sum_{2}^{m} c_{j}\left(x_{1}, x_{j}\right)$ $>c_{1}-\sum_{2}^{m} c_{j} / n \geqq c_{1}(1-(m-1) / n) \geqq 0$, a contradiction.

2.2 If $B$ is a finite set of unit vectors and $b$ is a number such that $b \geqq-1 / n$ and $(x, y)>b$ for all $x, y$ in $B$, there is a unit vector $z$ such that $(z, x)>$ $((1+(n-1) b) / n)^{\frac{1}{2}}$ for all $x$ in $B$.

We use only the case $b=-1 / n,((1+(n-1) b) / n)^{\frac{1}{2}}=1 / n$, but 2.2 in general is no more difficult to prove. Let $z$ be a unit vector that maximizes $\min _{x \in B}(z, x)$. If this maximum is $a$, than $a \geqq 0$ since 0 is not in the convex closure of $B$ by 2.1 (see [1, p. 9]). Further if $B_{1}$ is the subset of $B$ on which the maximum occurs, then 0 is in the convex closure of the projection of $B_{1}$ on the orthogonal complement of $z$, since otherwise there would be a unit vector $y$ orthogonal to $z$ such that $(y, x)>0\left(x \in B_{1}\right)$, and then the choice $z_{1}=(z+e y) /\left(1+e^{2}\right)^{\frac{1}{2}}$ (e positive, sufficiently small) would contradict the definition of $z$. Thus there is a relation $\sum_{1}^{m} c_{j} x_{j}=k z\left(c_{j}>0, \sum c_{j}=1, x_{j} \in B_{1}, m \leqq n\right)$. Taking the inner product of this equation with $z$ and then with $\sum x_{j}$, we get $k=a$ and then

$$
1+\sum_{j} c_{j} \sum_{i \neq j}\left(x_{i}, x_{j}\right)=m a^{2} \text {. }
$$

Since $\left(x_{i}, x_{j}\right)>b$, this yields $a^{2}>(1+(m-1) b) / m \geqq(1+(n-1) b) / n$, as required.

Now to start the proof of $1.2^{\prime}$, we assume there is no nonzero vector $z$ such that $(z, x) \geqq 0$ for all $x$ in $B$. This implies that 0 is in the convex closure of $B$, so that a nontrivial relation $\sum c_{j} x_{j}=0\left(c_{j}>0, x_{j} \in B\right)$ exists. If $l$ is a positive lower bound to the lengths of the elements of $B$, then by [5, Theorem 201] there exist positive integers $m_{j}$ and $m$ such that if $c_{j}=\left(m_{j}+r_{j}\right) / m$ then the $r_{j}$ are so small that $\left|\sum r_{j} x_{j}\right| \leqq 2 l / n$. Then $\sum m_{j} x_{j}$ is a nontrivial sum of elements of $B$, and its length is at most $2 l / n$. Let $\Sigma_{y_{j}}$ be such a sum with a minimum number of terms. Since $B$ has property $\mathrm{P}_{n},\left(y_{i}, y_{j}\right)>-1 / n\left|y_{i}\right|\left|y_{j}\right|$, all $i, j$, and thus by 2.2 there is a unit vector $z$ such that $\left(z, y_{j}\right)>\left|y_{j}\right| / n$, all $j$. But then $2 l / n \geqq\left|\Sigma y_{j}\right|$ $\geqq\left(z, \sum y_{j}\right)>\Sigma\left|y_{j}\right| / n \geqq 2 l / n$, a contradiction. Thus there is a nonzero vector $z$ such that $(z, x) \geqq 0$ for all $x$ in $B$, and we can complete the proof by induction on $n$, just as for 1.2 .

3. Proof of 1.4. The conclusion of 1.4 can be stated (and is proved) in the following form: if an integer $p(r)$ is associated with each $r$ in $S$ so that $p(-r)=-p(r)-1$ and the inequalities $(x, r)>p(r)(r \in S)$ are consistent, (that is, have a solution for $x)$, then these inequalities are a consequence of a subset of $n+1$ of them. 
Let $x=x_{0}$ be a solution of the inequalities and let $A$ be a minimal subset of elements of $S$ such that the inequalities $(x, a)>p(a)(a \in A)$ imply all inequalities. The proof that $A$ has $n+1$ elements is given in several steps.

(1) If $b \in A, r \in S, r-b \in S$ and $r$ is independent of $b$, then $p(r)=p(r-b)$ $+p(b)$. First note that $(x,-s)>p(-s)$ is equivalent to $(x, s)<p(s)+1$, for every $s$ in $S$. Thus $p(r-b)<\left(x_{0}, r-b\right)<p(r-b)+1, p(b)<\left(x_{0}, b\right)<p(b)+1$, by addition $p(r-b)+p(b)<\left(x_{0}, r\right)<p(r-b)+p(b)+2$, whence $p(r)=p(r-b)$ $+p(b)$ or $p(r)=p(r-b)+p(b)+1=p(b)-p(b-r)$. The last equation, however, yields a contradiction as follows. Because of the minimal nature of $A$, we can choose $x_{1}$ so that $\left(x_{1}, a\right)>p(a)(a \in A, a \neq b)$ and $\left(x_{1}, b\right)=p(b)$ (we can first achieve the inequality $\left(x_{1}, b\right) \leqq p(b)$, and then by moving $x_{1}$ towards $x_{0}$, the equality), and because $r$ is independent of $b$, so that also $\left(x_{1}, r\right) \neq p(r)$. Now at a point $x_{2}$ of the open segment $x_{0} x_{1}$ we have $\left(x_{2}, a\right)>p(a)$ for every $a$ in $A$, so that, by the definition of $A,\left(x_{2}, r\right)>p(r)$ and $\left(x_{2}, b-r\right)>p(b-r)$. If $x_{2}$ approaches $x_{1}$, this yields $\left(x_{1}, r\right) \geqq p(r)$ and $\left(x_{1}, b-r\right) \geqq p(b-r)$, whence $\left(x_{1}, r\right) \geqq$ $p(r)=p(b)-p(b-r) \geqq\left(x_{1}, b\right)-\left(x_{1}, b-r\right)=\left(x_{1}, r\right)$, so that $\left(x_{1}, r\right)=p(r)$, a contradiction.

(2) If $b$ and $c$ are in $A$ and independent, then $c-b \notin S$. For otherwise $p(b)=p(b-c)+p(c)$ and $p(c)=p(c-b)+p(b)$ by (1), then $0=-1$ by addition.

(3) If $x$ is a nonzero vector, there is $a$ in $A$ such that $(x, a)<0$. For, if $(x, a) \geqq 0$ for every $a$ in $A$, then $\left(x_{0}+k x, a\right)>n(a)$ for every positive number $k$, whence $\left(x_{0}+k x, r\right)>n(r)$ and $(x, r) \geqq 0$ for every $r$ in $S$. Since $-r \in S$, this yields $(x, r)=0$ and then $x=0$, a contradiction.

(4) $A$ is not the union of two nonempty subsets $B$ and $C$ which are orthogonal to each other. Assume the contrary. Since $S$ is indecomposable, there is $r$ in $S$ not orthogonal to $B$ or $C$. By (3) applied to the projection of $r$ on the subspace generated by $B$ there is $b_{1}$ in $B$ such that $\left(r, b_{1}\right)>0$, whence $r-b_{1}$ is in $S$. If $r-b_{1}$ is not orthogonal to $B$, then there is $b_{2}$ in $B$ such that $r-b_{1}-b_{2}$ is in $S$, and so on. This process can not continue indefinitely since there would then be a repetition $r-b_{1}-\ldots-b_{k}=r-b_{1}-\ldots-b_{m}(k<m)$, whence $p\left(r-b_{1}-\right.$ $\left.\ldots-b_{i}\right)=p\left(r-b_{1}-\ldots-b_{i+1}\right)+p\left(b_{i+1}\right)(k \leqq i<m)$ by $(1)$, and $p\left(b_{k+1}\right)+$ $\ldots+p\left(b_{m}\right)=0$ by addition, yielding the contradiction $0=\left(x_{0}, b_{k+1}+\ldots+b_{m}\right)$ $=\left(x_{0}, b_{k+1}\right)+\ldots+\left(x_{0}, b_{m}\right)>p\left(b_{k+1}\right)+\ldots+p\left(b_{m}\right)=0$. Thus for some $t, r-b_{1}$ $-\ldots-b_{t}$ is orthogonal to $B$. Starting with $s=-r+b_{1}+\ldots+b_{t-1}$, we can repeat the above procedure to get $c_{1}, c_{2} \ldots, c_{u}$ in $C$ such that $s-c_{1}, s-c_{1}-$ $c_{2}, \ldots, s-c_{1}-\ldots-c_{u}$ are all in $S$ and the last vector is orthogonal to $C$. But then $s-c_{1}-\ldots-c_{u}+b_{t}$ is orthogonal to $B$ and $C$, hence it is 0 by (3), so that $c_{u}-b_{t}=s-c_{1}-\ldots-c_{u-1}$, an element of $S$ in contradiction to (2).

(5) Completion of proof. Let $m$ be the number of elements of $A$. Since the region $(x, a)>p(a)(a \in A)$ is bounded, $m \geqq n+1$. Thus there is a linear relation among the elements of $A$, and in fact one of shortest nonzero length $q$, say 
$k_{1} a_{1}+\ldots+k_{q} a_{q}=0$. Clearly $q \leqq n+1$. If $r$ (resp. $s$ ) denotes the sum of the terms with positive (resp. negative) coefficients, then $(r, s) \geqq 0$ by $(2)$, whence $(r, r) \leqq(r, r+s)=0$, so that $r=0$ and all terms have the same sign which may be taken as positive. Now assume that $b$ is in $A$ and is not one of the $a_{j}$. Then $b$ is not a multiple of any $a_{j}$ since this would imply that $q=2$, that $b$ is a positive multiple of some $a_{j}$, and then that one of the inequalities $(x, b)>p(b),\left(x, a_{j}\right)>$ $p\left(a_{j}\right)$ is a consequence of the other, contradicting the minimality of $A$. By (4) we can choose $b$ to be nonorthogonal to some $a_{j}$; then $\left(b, a_{j}\right)<0$ by (2) and the fact that $S$ has property $\mathrm{P}$, so that $\left(b, a_{i}\right)>0$ for some $i$ since all coefficients in the above relation are positive, and $b-a_{i}$ is in $S$, contradicting (2). Thus $b$ does not exist, $m=q \leqq n+1, m=n+1$, and 1.4 is proved.

4. Proofs of 1.5 to 1.9 and related results. Assume that 1.5 is false, that $k$ is a positive integer such that $k r$ is in $S$ but $(k+1) r$ is not. Now $(5 r, r)>0$, whence $4 r$ is in $S$, and similarly so are $3 r$ and $2 r$. Thus $k \geqq 5$. By the assumptions on $S$, there is $s$ in $S$ such that $s$ is independent of $r$ and not orthogonal to $r$. Since $r$ may be replaced by $-r$, there is no restriction in assuming that also $(s, r)<0$, so that $s+r$ is in $S$. Then if $(s+r, r)<0, s+2 r$ is in $S$, and so on. After a finite number of steps we arrive at $t=s+j r$ in $S$ such that $-(r, r) \leqq(t, r)<0$. Then $t+k r$ and $t+2 r$ are in $S$, and since $(t+2 r,-r)<0$, so are $t-r$ and $t-2 r$. Now $(t+k r, t-r) \leqq 0$ since $(k+1) r$ is not in $S$; hence $(t+k r, t-2 r)<0$ and $2 t+(k-2) r$ is in $S$. This last vector, say $s_{1}$, is twice as far from the line of $r$ as $s$ (or $t)$ is, and it is not orthogonal to $r$ since $(2 t+(k-2) r, r)=2(t+r, r)+$ $(k-4)(r, r)>0$. Since the step from $s$ to $s_{1}$ can be repeated indefinitely, our original vector $s$ can be chosen so that $s$ (and hence $t$ ) is so far from the line of $r$ that $(t, t)>(2 k-1)(r, r)$. But now $(t+k r, t-r)=(t, t)+(k-1)(t, r)-$ $k(r, r)>(2 k-1)(r, r)-(k-1)(r, r)-k(r, r)=0$, so that $(k+1) r$ is in $S$, a contradiction. The second part of 1.5 is proved by the (unique) examples $S=$ $\{( \pm i, 0),( \pm j, \pm \sqrt{ } 6),(0, \pm 2 \sqrt{ } 6) ; 1 \leqq|i| \leqq 4,|j| \leqq 3\}$.

Next we prove 1.6. For convenience, we use multiple to mean nonzero integral multiple.

(1) There is an element of $S$ which has all its multiples in $S$. If $S$ is bounded, it has 0 as a point of accumulation: otherwise, there is a point of accumulation $x \neq 0$, then for elements $s$ and $t$ of $S$ which are distinct and close enough to $x$, $(s, t)>0, \mathrm{t}-s \in S$, and 0 is a point of accumulation anyway. Since $V$ can be covered by a finite number of cones with vertex 0 and verticle angle $\pi / 3$, there thus exists such a cone containing a sequence of distinct elements of $S$ tending to 0 or to $\infty$, and hence also containing elements $r$ and $s$ such that $|s|>8|r|$. It follows that $s-r, s-2 r, \ldots, s-5 r$ are all in $S$, the last inclusion, for example, coming from $(s-4 r, r)=(s, r)-4(r, r) \geqq 1 / 2|s||r|-4|r|^{2}>0$. Since

$$
(s, s-5 r)=(s, s)-5(s, r) \geqq|s|^{2}-5|s||r|>0,
$$

the vector $5 r$ belongs to $S$, and so do all multiples of $r$ by 1.5 . 
(2) If $s$ and $t$ are elements of $S$ such that all multiples of $s$ are in $S$ and $t$ is not orthogonal to $s$, then (a) all vectors $k s+t$ ( $k$ integer) are in $S \cup\{0\}$; (b) all multiples of $t$ are in $S$. Replacing $s$ by $-s$ if necessary, we may assume that $(s, t)<0$, whence $l s+t \in S \cup\{0\}$ for any positive integer $l$. Now if $l$ is large enough, $(s, l s+t)>0$, so that $(l-m) s+t \in S \cup\{0\}$ for any positive integer $m$, which is (a). Also for $l$ large enough $(l s+t,-l s+t)<0$, whence $2 t \in S$. Similarly $4 t, 8 t \in S$. But then $5 t \in S$, and (b) follows from 1.5 .

(3) If $r$ and $t$ are in $S$ and orthogonal, there is $s$ in $S$ not orthogonal to $r$ or $t$. Since $S$ is indecomposable, it contains a sequence $x_{1}, x_{2}, \ldots, x_{j}$ such that $x_{1}=r, x_{j}=t$, and consecutive terms are not orthogonal. If a shortest such sequence is chosen, then nonconsecutive terms are orthogonal, and $j=3$, since otherwise the sequence could be shortened by replacing the pair $x_{2}, x_{3}$ by $x_{2}+x_{3}$ if $\left(x_{2}, x_{3}\right)<0$ or by $x_{2}-x_{3}$ if $\left(x_{2}, x_{3}\right)>0$. The choice $s=x_{2}$ yields (3).

(4) If $r, t \in S, r \neq t$, then $r-t \in S$. First note that all multiples of all elements of $S$ are again in $S$ by (1), (2b) and (3). Thus if $r$ is not orthogonal to $t, r-t \in S$ by (2a); while if $r$ is orthogonal to $t$, there is $s \in S$ not orthogonal to $r$ or $t$ by (3), then $k s+t, k s+r \in S$ for all integers $k$ by $(2 \mathrm{a})$, and, since $(k s+t, k s+r)>0$ for $k$ large enough, $r-t \in S$ in this case also.

By (4), $S \cup\{0\}$ is closed under subtraction, hence is a group, and 1.6 is proved.

From 1.6 we get 1.7. The nonzero real (or rational) numbers of absolute value less than 1 show that neither of these results is true if $n=1$.

Since the only if part of 1.8 is easily verified, we turn to the if part. If $n \geqq 2$, $S \cup\{0\}$ is a group by 1.6, and since it is discrete (because 0 is not a point of accumulation) and contains $n$ independent elements, it must be an $n$-dimensional lattice. If $n=1$, it is easily proved that $S$ has a smallest element $r$ and consists of the multiples of $r$.

To start the proof of 1.9, assume $u, v \in S_{1}, u \neq-v$ and $(u, v)<0$. Then there are scalars $k, l$ such that $u+k s, v+l s \in S$. Now if $k>0$, then $(u+k s, s)>0$ and $u+k s$ can be replaced by $u+(k-1) s$ which is also in $S$. Repeating this procedure as often as necessary, we may assume $k \leqq 0$, and similarly $l \geqq 0$. Then $(u+k s, v+l s)=(u, v)+k l(s, s)<0, u+v+(k+l) s \in S$, and $u+v \in S_{1}$. Thus $S_{1}$ has property P. Now let $Q$ and $R$ be two lower-dimensional orthogonal sunspaces of $s^{0}$. Since $S$ is indecomposable, then by the same reasoning as in step (3) of the proof of 1.6, there is $x$ in $S$ orthogonal to neither $Q$ nor $R$. Since $x$ projects onto an element of $S_{1}$ not in $Q$ or $R$ and the pair $Q, R$ is arbitrary, $S_{1}$ is indecomposable.

We can now refine 1.7 .

4.1. Let $S$ be symmetric, indecomposable, have property $\mathrm{P}$ and contain $r$. In the respective cases that the dimension $n$ of $S$ is 2,3 , at least 4 , assume that $S$ contains $5 r, 4 r, 3 r$. Then $S \cup\{0\}$ is a group. If a smaller positive multiple 
of $r$ is assumed to be in $S$, then $S \cup\{0\}$ need not be a group, in fact need not be infinite.

If $n=2$, we use 1.7. Assume $n=3$. By the methods used at the beginning of the proof 1.5, there is $s$ in $S$ such that $s$ is independent of $r$, and $s+r, s+2 r, \ldots$, $s+5 r$ are all in $S$. The projection of $S$ on $s^{0}$ contains $r_{1}$, the projection of $r$, and also $5 r_{1}$. By 1.9 and 1.5 we conclude that $S$ is infinite, and then by 1.6 that $S \cup\{0\}$ is a group. The corresponding proof for $n \geqq 4$ is similar. To prove the final part of 4.1 , we add to the example used in the proof of 1.5 two further examples: if $n=3, S=\{(i, 0,0),(j, \pm \sqrt{ } 3, \pm 1),(j, 0, \pm 2),(0, \pm 2 \sqrt{ } 3),(0, \sqrt{ } 3, \pm 3)$; $1 \leqq|i| \leqq 3,|j| \leqq 2\}$, and if $n \geqq 4$ (in fact, if $n$ is arbitrary), $S=\left\{\left(x_{1}, x_{2}, \ldots, x_{n}\right)\right.$; $x_{j}$ an integer, $\Sigma\left|x_{j}\right|=1$ or 2$\}$.

\section{REFERENCES}

1. T. Bonnesen and W. Fenchel, Theorie der konvexen Koerper, Springer, Berlin, 1927; or New York, Chelsea, 1948.

2. A. Borel and F. Hirzebruch, Characteristic classes and homogeneous spaces. I, Amer. J. Math. 80 (1958), 458-538.

3. E. Cartan, Complément au mémoire "sur la géométrie des groupes simples", Ann. Mat. 5 (1928), 253-260.

4. E. B. Dynkin, The structure of semi-simple Lie algebras, Amer. Math. Soc. Transl. (2) 17 (1950).

5. G. H. Hardy and E. M. Wright, Theory of numbers, 4th ed., Oxford, 1960.

6. Harish-Chandra, Representations of semisimple Lie groups. IV, Amer. J. Math. 77 (1955), 743-777.

UNIVERSITy OF CALIFoRnia,

Los Angeles, California 